https://doi.org/10.37208/tgn27209

\section{How not to be the perfect host: a simple tactic for avoiding sheep ticks (Ixodes ricinus)}

\author{
I.C. Wilkie
}

Institute of Biodiversity, Animal Health and Comparative Medicine, University of Glasgow, Glasgow G12 8QQ

E-mail: iain.wilkie@glasgow.ac.uk

Ticks (Arachnida: Ixodida) are external parasites that require a blood meal at various stages of their life-cycle. The sheep tick (Ixodes ricinus) has been described as "one of the most dangerous animals in Europe" because whilst feeding it can transmit a range of pathogens that cause human diseases such as Lyme borreliosis (Lyme disease) and tick-borne encephalitis (Rahlenbeck et al., 2016). Scotland has the highest incidence of Lyme disease in the U.K. (27\% of all U.K. cases in 2001-2012: Cairns et al., 2019) and this is predicted to increase as a result of changing climatic conditions (Li et al., 2016). This note describes a simple method for reducing the risk of becoming a tick host and provides preliminary information on its effectiveness.

Sheep ticks employ a host-seeking strategy known as "vantage point host-ambushing": when environmental conditions are suitable they leave the ground microhabitat of leaf litter and climb up available vegetation such as rush or grass stems (Carr \& Salgado, 2019). They then stop, cling to the vegetation using their second to fourth pairs of legs, and adopt one of two postures: in the questing posture the first two legs are extended to the front, either immobile or waving actively (Fig. 1); in the reposing posture the forelegs are folded (Lees, 1948). Tick forelegs are important both for sensing an approaching host, which depends mainly on the detection of chemical and thermal stimuli by the Haller's organs located on these legs (Carr \& Salgado, 2019), and for attaching to the host by means of their terminal claws (Voigt \& Gorb, 2017).

The following observations were made between 21 st April and 28th May 2019 in Strachur Estate, Cowal, Argyll, Scotland in rough grassland dominated by the tussock-forming purple moor-grass (Molinia caerulea) (Fig. 2). When walking over such grassland it was noticed that sweeping the vegetation ahead with a stout wooden stick (normally used as a walking aid) tended to cause perching ticks to drop to the ground, thereby interrupting their host-seeking behaviour. The effectiveness of this method was then assessed by recording the effect on ticks of semi-standardised mechanical disturbance using the stick. Thirty ticks

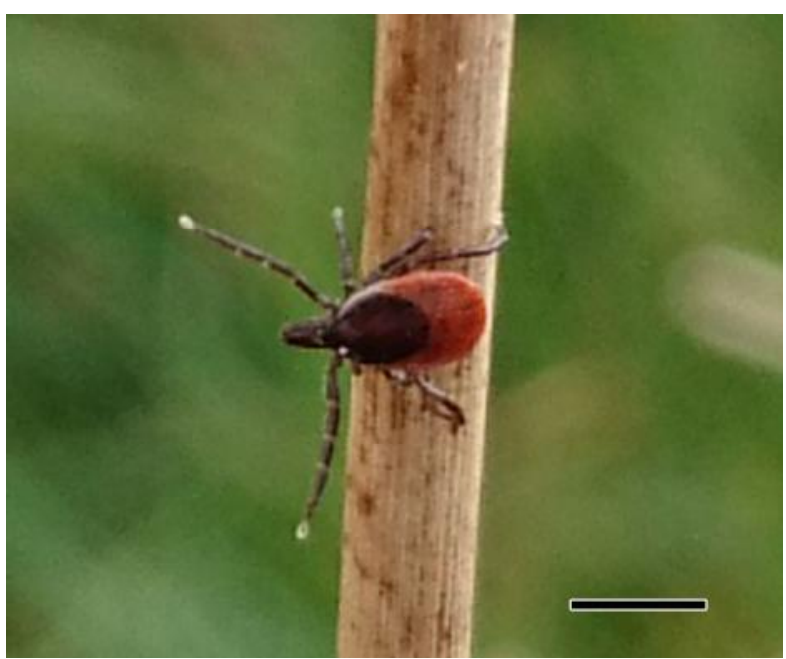

Fig. 1. A sheep tick (Ixodes ricinus) on a withered rush stem (Juncus sp.). The tick is demonstrating the questing posture. Photographed in Strachur Estate, Argyll, Scotland on 2nd May 2019. Scale bar $=2 \mathrm{~mm}$. (Photo: I.C. Wilkie)

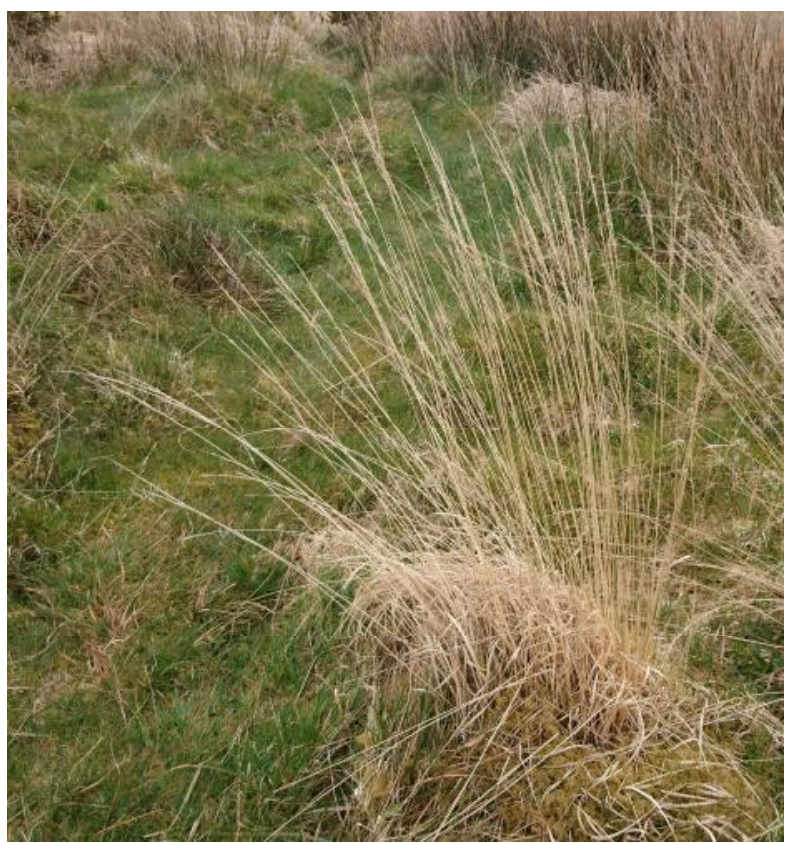

Fig. 2. Withered purple moor-grass (Molinia caerulea) at a location where the observations were made. Photographed in Strachur Estate, Argyll, Scotland on 21st April 2019. (Photo: I.C. Wilkie)

including adults and nymphs (immature stages) were observed. All were located near the tips of withered $M$. caerulea seed-heads that were leaning towards or over established routes used by foxes (Vulpes vulpes), roe deer (Capreolus capreolus), and red deer (Cervus elaphus), as deduced from the presence of their respective droppings and/or tracks. Ticks are more easily seen in April and May because the previous year's grasses are maximally bleached and there is little new growth. When a tick was spotted, the height of its vantage point was measured and then the grass stem was hit repeatedly with the stick (length $80 \mathrm{~cm}$; diameter $c a$. $2.5 \mathrm{~cm}$ ) roughly halfway between the vantage point and 
the ground and at a frequency of one hit per second; only light force was used - enough to deflect the grass stem without damaging it. The number of hits applied before the tick dropped was recorded.

The height of the tick vantage points was $42-160 \mathrm{~cm}$ (mean $78 \mathrm{~cm}$ ). Fig. 3 shows that 25 ticks (83\%) dropped after one to three hits; three ticks (10\%) dropped after more than three (up to nine) hits; and two ticks (7\%) could not be dislodged at all, even when the seed-head was nipped off and "flicked" repeatedly with an index finger. Since $83 \%$ of ticks dropped after their vantage point vegetation was hit one to three times, these results suggest that beating the vegetation ahead lightly with a stick or walking pole around once per second could reduce significantly the risk of being ambushed by ticks. A full-scale experimental investigation would be needed to confirm this and determine if such a tactic should be added to the list of safety measures already recommended to the public for avoiding ticks (see e.g. NHS, 2019).

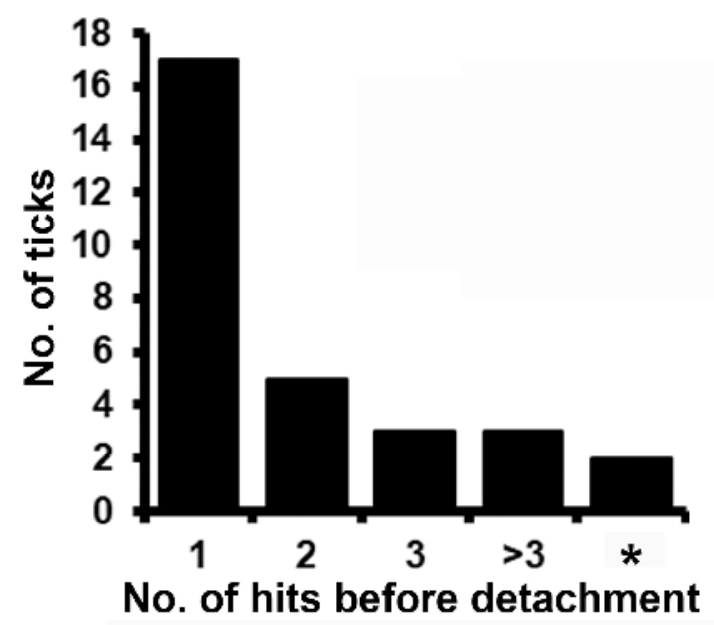

Fig. 3. Effect on 30 sheep ticks of semi-standardised mechanical disturbance. *No detachment.

The biological significance of these observations is not clear. A key question is: does the impact of the stick simply knock a tick off its perch because its grip is not tight enough (i.e. no behavioural reaction is involved), or does the tick react to the impact by releasing its grip? The latter would be an example of a dropping response. Many animals exhibit dropping responses whereby they fall suddenly under the influence of gravity alone, usually as a defensive strategy for avoiding predation (Humphreys \& Ruxton, 2019; Wilkie, 2019). Although there appear to be no reports in the literature that external stimuli such as mechanical disturbance invoke a dropping response in sheep ticks, it is well known that they release their grip and drop from their host when they have completed their blood meal, and Lees (1948) noted that in laboratory conditions a questing tick that attaches itself to "neutral" objects like cotton wool "soon loses interest and drops off". It seems likely that the effect of mechanical disturbance on ticks described in this note involved a dropping response in at least some cases. Of the 28 ticks that dropped, 11 (39\%) did so after more than one hit and therefore their grip was initially tight enough to resist dislodgement. Since the force applied at each hit was roughly the same, this implies that after one or more hits, a behavioural reaction - grip loosening - was instigated. Such a dropping response could be primarily defensive, for example enabling ticks to avoid incidental ingestion by larger grazing herbivores (Gish et al., 2011), or perhaps more likely it could be a strategy for bringing ticks into contact with medium-sized mammals such as foxes passing below the level of their vantage points.

I am grateful to Keith Watson for grass identification and to a reviewer for helpful comments.

\section{REFERENCES}

Cairns, V., Wallenhorst, C., Rietbrock, S. \& Martinez, C. (2019). Incidence of Lyme disease in the UK: a population-based cohort study. British Medical Journal Open 9:e025916. https://doi.org/10.1136/bmjopen-2018-025916

Carr, A.L., \& Salgado, V.L. (2019). Ticks home in on body heat: a new understanding of Haller's organ and repellent action. PLoS ONE 14(8): e0221659. https://doi.org/10.1371/journal.pone.0221659

Gish, M., Dafni, A. \& Inbar, M. (2011). Avoiding incidental predation by mammalian herbivores: accurate detection and efficient response in aphids. Naturwissenschaften 98, 731-738. https://doi.org/10.1007/s00114-011-0819-7

Humphreys, R.K. \& Ruxton, G.D. (2019). Dropping to escape: a review of an under-appreciated antipredator defence. Biological Reviews 94, 575-589. https://doi.org/10.1111/brv.12466

Lees, A.D. (1948). The sensory physiology of the sheep tick, Ixodes ricinus L. Journal of Experimental Biology 25, 145-207.

Li, S., Gilbert, L., Harrison, P.A. \& Rounsevell, M.D.A. (2016). Modelling the seasonality of Lyme disease risk and the potential impacts of a warming climate within the heterogeneous landscapes of Scotland. Journal of the Royal Society Interface 13: 20160140. https://doi.org/10.1098/rsif.2016.0140

National Health Service (NHS) (2019). https://www.nhs.uk/conditions/lyme-disease/ Accessed 24th October 2019.

Rahlenbeck, S., Fingerle, V. \& Doggett, S. (2016). Prevention of tick-borne diseases: an overview. British Journal of General Practice 66, 492-494. https://doi.org/10.3399/bjgp16X687013

Voigt, D. \& Gorb, S. (2017). Functional morphology of tarsal adhesive pads and attachment ability in ticks Ixodes ricinus (Arachnida, Acari, Ixodidae). Journal of Experimental Biology 220, 1984-1996. https://doi.org/10.1242/jeb.152942

Wilkie, I.C. (2019). Defensive behaviour in the scotch argus Erebia aethiops (Lepidoptera: Nymphalidae). The Glasgow Naturalist 27(1), 81-82. 